\title{
Combination of doxorubicin liposomes with left atrial appendage radiofrequency catheter ablation to reduce post-ablation recovery of electrical conduction
}

This article was published in the following Dove Medical Press journal:

International Journal of Nanomedicine

\author{
Ying Zhuge ${ }^{1, *}$ \\ Huan-Er Ni ${ }^{1, *}$ \\ Yu-Jie Wang ${ }^{2}$ \\ $\mathrm{Mu}-\mathrm{Ye} \mathrm{He}^{2}$ \\ Jun-Shan Wang ${ }^{3}$ \\ Feng Gao 2,4 \\ Fang Wang'
}

'Department of Cardiology, Shanghai General Hospital, Shanghai Jiao Tong University School of Medicine, Shanghai 200800, People's Republic of China; ${ }^{2}$ Department of Pharmaceutics, School of Pharmacy, East China University of Science and Technology, Shanghai 200237, People's Republic of China; ${ }^{3}$ Department of Gastroenterology, Shanghai Tenth People's Hospital, Tongji University, Shanghai 200072, People's Republic of China; ${ }^{4}$ Shanghai Key Laboratory of Functional Materials Chemistry, East China University of Science and Technology, Shanghai 200237, People's Republic of China

*These authors contributed equally to this work
Correspondence: Fang Wang Department of Cardiology, Shanghai General Hospital, Shanghai Jiao Tong University School of Medicine, 100 Haining Road, Hongkou District, Shanghai 200080, People's Republic of China

Tel +862163240090

Fax +86 2I 63240090

Email onlyfangl@hotmail.com
Aims: To determine whether use of radiofrequency catheter ablation (RFCA) combined with intravenously administered liposomal doxorubicin (L-DOX) facilitates a reduction in the recovery of post-ablation electrical conduction.

Methods: Circumferential ablation was performed on the epicardial surface of the left atrial appendage (LAA) in New Zealand White rabbits, and L-DOX was then administered intravenously. Fluorescence spectrophotometry was used to assess reagent bio-distribution, while Western blots and immunohistochemistry were used to assess the localization of the apoptotic markers Bcl-2, Bax, and cleaved CASP 3 in the LAA. Liver, kidney, and cardiac functions were also measured to evaluate the safety of this approach.

Results: At 1 week and 1 month after RFCA, a pacing electrocardiogram could not be detected in most of the rabbits that had received the combined RFCA and L-DOX therapy. L-DOX began to target the LAA on the second day after RFCA. L-DOX treatment increased the apoptosis of cardiomyocytes in the regions peripheral to the necrotic area induced by RFCA. Doxorubicin had some effect on liver and kidney function, but these effects were reversible and did not affect survival.

Conclusion: The present results provide evidence that L-DOX treatment can reduce the recovery of electrical conduction after RFCA therapy owing to L-DOX-induced apoptosis of cardiomyocytes in the ablated area and the proximal transition zone of the LAA.

Keywords: doxorubicin, liposome, radiofrequency catheter ablation, atrial fibrillation, recovered electrical conduction

\section{Introduction}

Atrial fibrillation (AF) is the most common form of arrhythmia and is associated with high morbidity and mortality. ${ }^{1}$ However, current pharmaceutical approaches are generally not sufficient to treat this condition, owing to recurrent AF episodes and side effects associated with long-term treatment. Over the last decade, radiofrequency catheter ablation (RFCA) has been established as the preferred treatment for a subset of patients with symptomatic AF, offering these individuals clear improvements in their quality of life. ${ }^{2}$ RFCA approaches such as pulmonary vein isolation are effective treatments for drug-refractory AF. ${ }^{1,3-5}$ However, while RFCA treatment induces acute coagulation and necrosis of myocardial cells at the localized site where high temperature is initially applied, peripheral myocardial cells can undergo sub-lethal and reversible damage owing to lower temperatures, enabling some of these cells to recover 
over time. As a result, incomplete ablation coupled with the recovery of acutely damaged myocardial cells can contribute to a recovery of electrical conduction at the ablated site..$^{6-9}$

RFCA is known to induce an inflammatory reaction. ${ }^{10}$ According to a previous study, doxorubicin (DOX) is one of the most widely used clinical anthracycline anti-tumor drugs ${ }^{11}$ and liposomes have inflammation-targeting properties. ${ }^{12}$ In our project, liposomal doxorubicin (L-DOX) were injected into the auricular veins of rabbits after circumferential ablation of the anterior and posterior wall of the left atrial appendage (LAA), which is a source of localized re-entrant tachycardia and an important non-pulmonary vein trigger of AF. ${ }^{13-15}$ We found that L-DOX could effectively reach the LAA ablation site owing to its inflammation-targeting capabilities, and once there, it could completely kill acutely damaged myocardial cells that would otherwise be able to recover, thus maximizing the therapeutic efficacy of RFCA (Figure 1).

\section{Methods}

\section{RFCA operation procedures}

The operation was performed using aseptic techniques with sterile instruments. Before surgery, rabbits were injected with $1 \%$ pentobarbital sodium that was administered into the auricular vein at a dose of $3 \mathrm{~mL} / \mathrm{kg}$. After the corneal reflex was no longer apparent, rabbits were placed on a warming operative platform $\left(37^{\circ} \mathrm{C}\right)$ in the supine position. The third and fourth left ribs were cut, the chest was opened, and the whole heart was then exposed using a chest expander. An incision was made in the pericardium to expose the myocardial wall, and the LAA was gently exteriorized using smooth forceps and a medical suture line was threaded through the distal end of the LAA.

Before ablation, the following parameters were set on the Cardiac Ablation Generator (IBI-1500T8, Irvine Biomedical Inc., Irvine, CA, USA): power $=15 \mathrm{~W}$, temperature $=55^{\circ} \mathrm{C}$, time $=15$ seconds. The LAA was then slightly raised using the medical suture line, and a $4 \mathrm{~mm}$ quadrupole temperaturecontrolled ablation catheter (Celsius, Biosense Webster Inc., Irwindale, CA, USA) was used to perform circumferential ablation around the anterior and posterior wall of the LAA (Figure S1). The number of ablation lesions or the distance between lesions was not pre-defined. RFCA was conducted until a bidirectional conduction block of the LAA was achieved. As such, the empirical electrical isolation of the LAA was the endpoint of this ablation procedure.

\section{Fluorescence imaging}

IR-775 chloride, a near-infrared fluorescent dye with an excitation wavelength of $740 \mathrm{~nm}$ and an emission wavelength of $790 \mathrm{~nm}$, was used to reveal in vivo agent distributions.
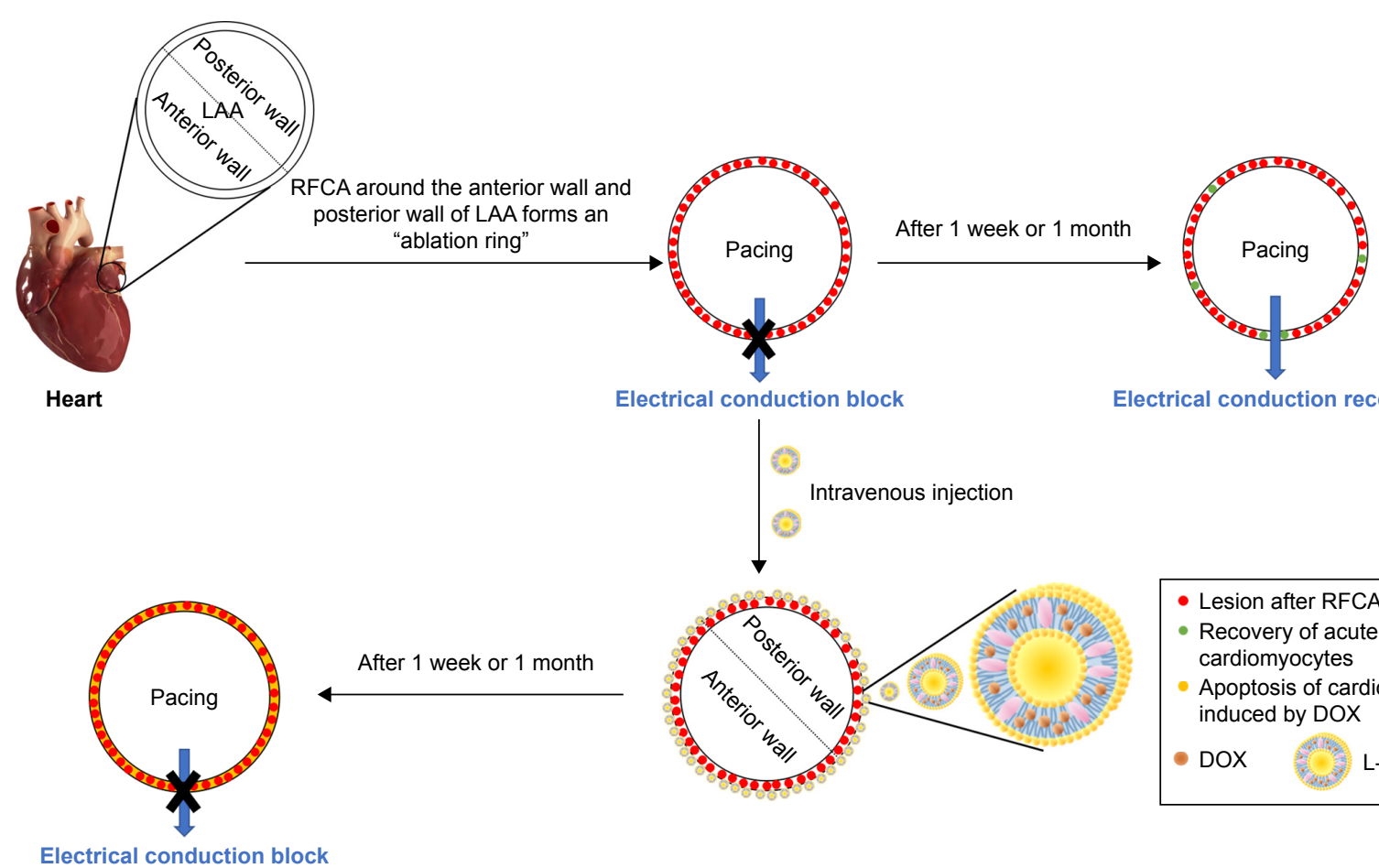

ectrical conduction block

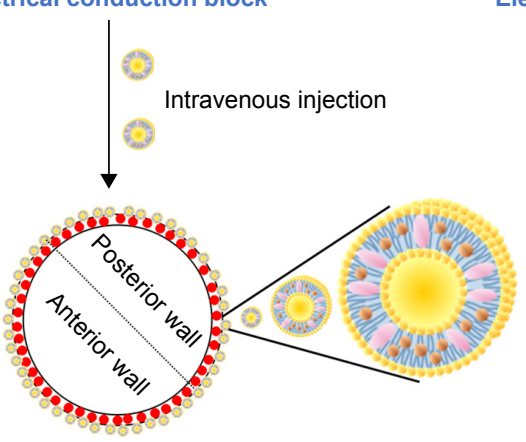

Electrical conduction recovery
- Lesion after RFCA

- Recovery of acute necrotic cardiomyocytes

- Apoptosis of cardiomyocytes induced by DOX

- DOX L-DOX

Figure I Illustration of the use of L-DOX in combination with LAA RFCA to reduce the recovery of electrical conduction. Abbreviations: L-DOX, liposomal doxorubicin; LAA, left atrial appendage; RFCA, radiofrequency catheter ablation. 
The IR-775 liposome (L-IR-775) preparation protocols are available in the online Supplementary material. Twenty-four adult New Zealand White rabbits were randomly divided into four groups: group A, blank; group B, intravenous administration of L-IR-775 (0.5 mg IR-775/kg) after thoracotomy without RFCA (sham-operated [SO]+L-IR-775 group); group $\mathrm{C}$, intravenous administration of free IR-775 (0.5 mg/kg) after RFCA (RFCA+IR-775 group); group D, intravenous administration of L-IR-775 (0.5 mg IR-775/kg) after RFCA (RFCA+L-IR-775 group). Group D was subdivided into two groups in which the rabbits were sacrificed after 24 hours and 48 hours respectively. Free IR-775 and L-IR-775 were administered as a single dose through intravenous auricular vein administration.

Organs of interest (including heart, liver, spleen, lung, and kidney) were harvested, rinsed with saline, and placed in a culture dish. This culture dish was placed in an In-vivo Imaging System (Lumina III, PerkinElmer Inc., Waltham, MA, USA) equipped with a fluorescent filter $\left(\lambda_{\text {ex }}=740 \mathrm{~nm}, \lambda_{\mathrm{em}}=790 \mathrm{~nm}\right)$. The settings were as follows: subject height $=1.5 \mathrm{~cm}$, near infrared excitation light intensity $=8 \mathrm{~mW} / \mathrm{cm}^{2}$, pixel resolution $=1,024 \times 1,024$, exposure time $=1$ second, binning $=2 \times 2$.

\section{Pacing and mapping maneuver}

The L-DOX preparation protocols are available in the online Supplementary material. Thirty adult New Zealand White rabbits were randomly divided into three groups: group A, RFCA; group $\mathrm{B}$, intravenous administration of free DOX (4 mg/kg) after RFCA (RFCA+DOX (4 mg/kg) group); group $\mathrm{C}$, intravenous administration of $\mathrm{L}-\mathrm{DOX}$ (4 mg DOX $/ \mathrm{kg}$ ) after RFCA (RFCA+L-DOX (4 mg DOX/kg) group). Free DOX and L-DOX were administered as a single intravenous dose in the auricular vein.

Electrical conduction between the interior and exterior circumferential ablation lesions was assessed in all animals before ablation, as well as 5 minutes, 1 week, and 1 month after RFCA. The rabbits' chests were closed, and wounds were sutured and disinfected 5 minutes after RFCA. After waking up, the animals were sent to the laboratory animal center for feeding. All animals received a dose of 400,000 U/day of penicillin intramuscularly for 3 consecutive days. One week and 1 month after the surgery, each rabbit's chest was reopened and a pacing electrode was placed on the epicardium to detect the pacing electrocardiogram.

The electrocardiogram was monitored and stored on a BL420 Biological Signal Acquisition and Analysis System (BL420S, Taimeng Software Co. Ltd., Chengdu, People's
Republic of China). The tip of the stimulating electrode was attached to the anterior or posterior wall of the LAA inside the circumferential ablation lesion (Figure S2) and it was connected to the Cardiac Electrophysiological Stimulator (CES, DF-5A, Suzhou Dongfang Electronic Instrument Factory, Suzhou, People's Republic of China). Rapid S1S1 (150 ms cycle length) procedural stimuli were adapted to pace.

The myocardial electrogram was used to reflect the muscle excitatory activity of the LAA muscle to verify whether it was captured by the pacing signal (Figures S3 and $\underline{\mathrm{S} 4}$ ). The electrical conduction block is available in the online Supplementary material (Figure S5). Successful LAA conduction block was defined as no detection of pacing electrocardiogram while pacing myocardial tissues inside the circumferential ablation lesions.

\section{L-DOX tissue distribution}

Twenty-five adult New Zealand White rabbits were randomly divided into two groups: group $A(n=5)$, $\mathrm{RFCA}+\mathrm{DOX}(4 \mathrm{mg} / \mathrm{kg})$; group B $(\mathrm{n}=20)$, RFCA+L-DOX (4 mg DOX/kg).

Five rabbits in group A were sacrificed on the second day after intravenous injection. Twenty rabbits in group B were randomly divided into four smaller groups, which were sacrificed on the first, second, fifth, and seventh day after intravenous injection. Key organ samples (including heart, liver, spleen, lung, and kidney) were harvested and stored at $-80^{\circ} \mathrm{C}$. The concentration of DOX in tissues was measured by fluorescence spectrophotometry with a fluorescence analysis spectrometer (Lumina, Thermo Fisher Scientific, Waltham, MA, USA), following previously published protocols $^{16,17}$ (the specific detection steps are available in the online Supplementary material).

\section{Detection of LAA cardiomyocyte apoptosis}

Fifteen adult New Zealand White rabbits were randomly divided into three groups: group $\mathrm{A}, \mathrm{SO}$; group $\mathrm{B}, \mathrm{RFCA}$; group C, RFCA+L-DOX (4 mg DOX/kg).

After 1 week, rabbits were sacrificed, hearts were collected and the LAA was carefully cut out. The LAA was stored in normal saline in order to remove blood, and was then fixed in $4 \%$ paraformaldehyde and frozen at $-80^{\circ} \mathrm{C}$ until further analysis. TUNEL staining was used to assess cardiomyocyte apoptosis in the ablated area and in the transition zone of the LAA (the specific detection steps are available in the online Supplementary material). 


\section{Western blot analysis of LAA proteins}

The expression of Bcl-2, Bax, and cleaved CASP3 in the ablated area and transition zone of the LAA were detected by Western blotting. Fifteen adult New Zealand White rabbits were randomly divided into three groups: group $\mathrm{A}, \mathrm{SO}$; group B, RFCA; group C, RFCA+L-DOX (4 mg DOX/kg).

After 1 week, rabbits were sacrificed and the LAA was collected as previously described. We then used SDS-PAGE to analyze proteins. Those proteins were electro-transferred to PVDF membranes after electrophoresis. These membranes were used for immunoblotting with appropriate antibodies. The protein bands were scanned and quantified by using ImageJ software (specific detection steps are available in the online Supplementary material).

\section{Semi-quantitative immunohistochemical (IHC) examination}

A total of 15 adult New Zealand White rabbits were randomly divided into three groups: group A, SO; group B, RFCA; group C, RFCA+L-DOX (4 mg DOX/kg).

After 1 week, rabbits were sacrificed and LAA specimens were collected. The expression of Bcl-2, Bax, and cleaved CASP3 was assessed by IHC staining using appropriate antibodies (specific detection steps are available in the online Supplementary material).

\section{Evaluation of kidney and liver function}

Twenty adult New Zealand White rabbits were randomly divided into four groups: group A, SO; group B, RFCA; group C, RFCA+L-DOX (3 mg DOX/kg); group D, RFCA+L-DOX (4 mg DOX/kg).

On the first, third, fifth, and seventh day after intravenous injection, a blood sample $(1 \mathrm{~mL})$ was collected from the auricular vein. These blood samples were centrifuged at $5,000 \mathrm{rpm} / \mathrm{min}$ for 15 minutes, and serum was collected and stored at $-20^{\circ} \mathrm{C}$ until further analysis.

Using respective colorimetric diagnostic kits (Nanjing Jiancheng Bioengineering Institute, Nanjing, People's Republic of China), the levels of serum ALT and AST were determined to evaluate liver function and hepatotoxicity, while blood urea nitrogen (BUN) and serum creatinine were measured to evaluate renal function and nephrotoxicity. The results were calculated as instructed by the manufacturers.

\section{Echocardiography determination}

Fifteen adult New Zealand White rabbits were randomly divided into three groups: group A, SO; group B, RFCA; group C, RFCA+L-DOX (4 mg DOX/kg).
After 1 week, rabbits were subjected to induction of anesthesia at a concentration of $4 \%$ and were maintained in a deeply anesthetized state with $2 \%$ isoflurane.

We invited two experienced ultrasonographic doctors who were blinded to the grouping of rabbits to perform transthoracic echocardiography. The left ventricular ejection fraction (LVEF), left ventricular fraction of shorting (LVFS), left ventricular end diastolic diameter (LVEDD), stroke volume, left ventricular posterior wall depth (LVPWD), and left ventricular end diastolic volume (LVEDV) of each rabbit were assessed using a $12 \mathrm{MHz}$ transducer connected to a commercially available echocardiographic system (Phillips Sonos 7500, Amsterdam, Holland). All measurements are averages from three consecutive cardiac cycles.

\section{Statistical analysis}

IBM SPSS Statistics 22.0 software was used for all statistical analyses, and data were expressed as means \pm SD based on three to six independent experiments. Paired Student's $t$-tests were used to evaluate statistical significance. A $P$-value of less than 0.05 was considered statistically significant.

\section{Ethics statement}

All animal experiments were approved by the Laboratory Animal Center, Shanghai Tenth People's Hospital, and all animal procedures were in accordance with the guidelines from Directive 2010/63/EU of the European Parliament on the protection of animals used for scientific purposes. For all in vivo experiments, adult New Zealand White rabbits (4-6 months old, 2.2-2.8 kg body weight) purchased from Shanghai Shengwang Experimental Animal Breeding Co. Ltd. (Shanghai, People's Republic of China) were used. Male and female adult New Zealand White rabbits were maintained in a temperature-controlled $\left(25^{\circ} \mathrm{C}\right)$ facility with a 12-hour light/dark cycle. Figure $\mathrm{S} 6$ shows the number and group assignment of the animals.

\section{Results}

\section{The circumferential RFCA lesions}

Upon completion of the ablation procedure, the circumferential ablation lesions were visible. There were approximately 5-6 ablation points in the anterior wall of the LAA, and approximately 4-5 ablation points in the posterior wall. The lesions associated with each ablation point were continuous and without gaps. The ablation points were coupled to each other to form a complete closed annular ablation ring, which passed through the anterior and posterior walls and 

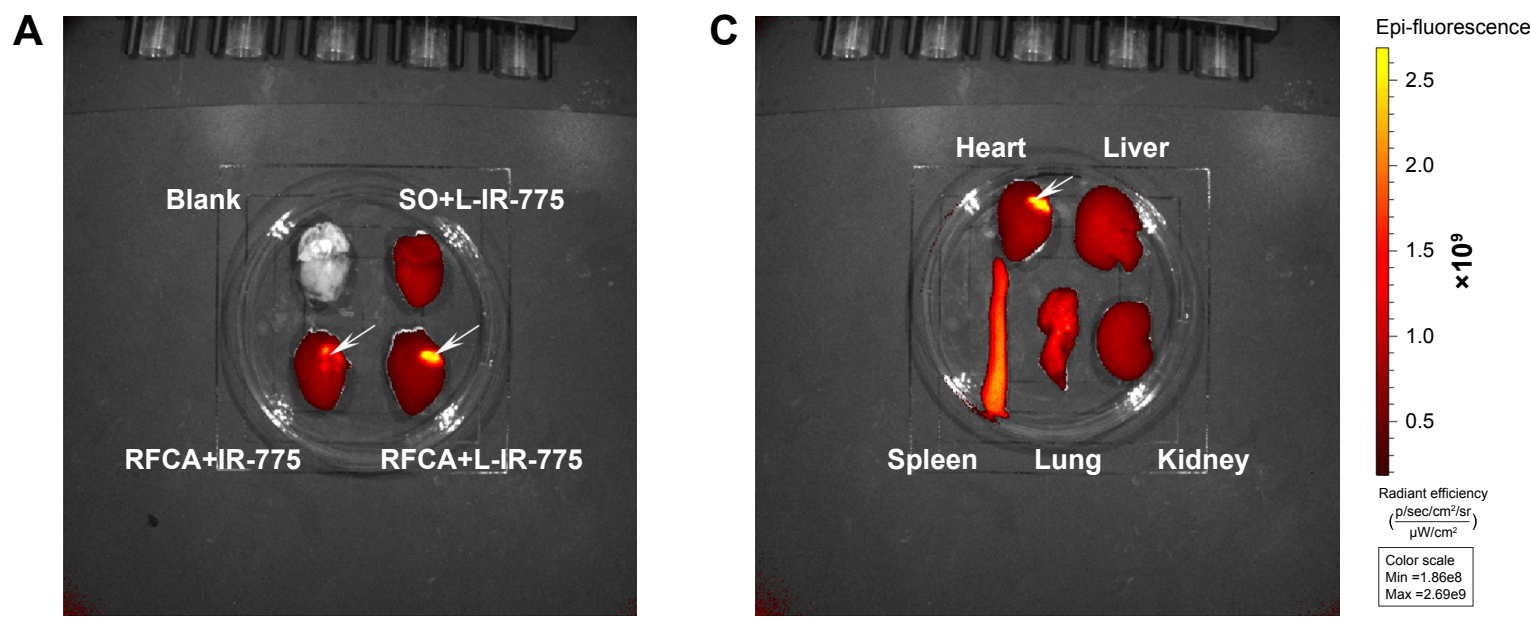

B

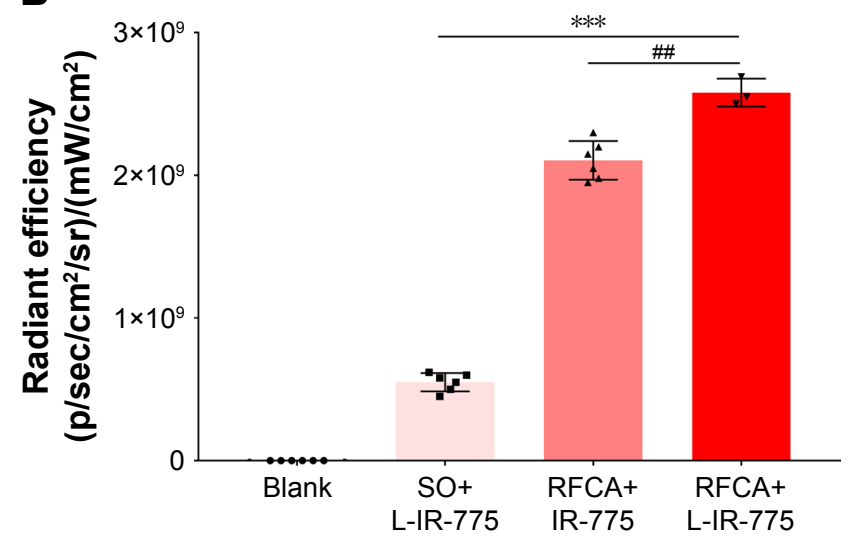

D

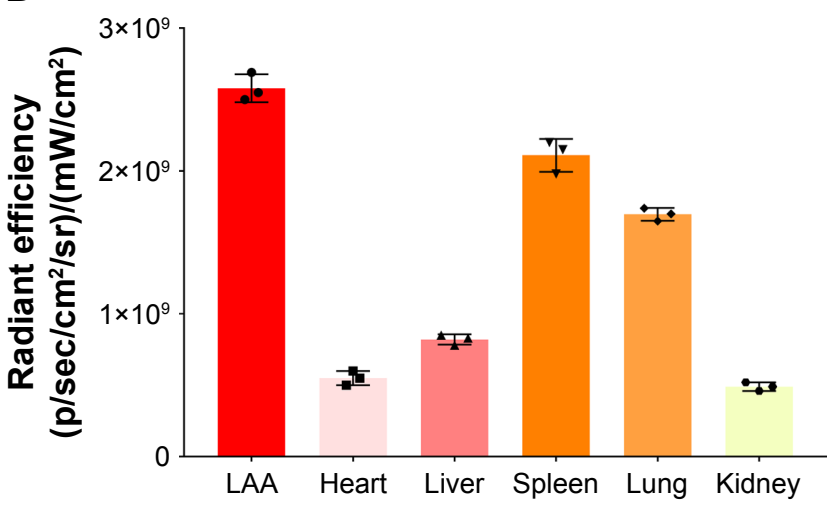

Figure 2 Fluorescence imaging and semi-quantitative data from rabbits following group-appropriate treatments.

Notes: Fluorescence imaging (A) and semi-quantitative data (B). Group A, blank; Group B, SO+L-IR-775; Group C, RFCA+IR-775; Group D, RFCA+L-IR-775. All rabbits were sacrificed 48 hours after intravenous drug administration. Fluorescence imaging (C) and semi-quantitative data (D) from tissues of rabbits in Group $D$. The white arrow indicates the LAA ablation site. ${ }^{* * *} P<0.00$ I versus SO+L-IR-775 treatment; ${ }^{*} P<0.01$ vs RFCA+IR-775 treatment. Data are presented as means \pm SD.

Abbreviations: LAA, left atrial appendage; L-IR-775, IR-775 liposome; RFCA, radiofrequency catheter ablation; SO, sham-operated.

surrounded the root of the LAA to achieve empirical LAA electrical isolation.

\section{Fluorescence imaging}

L-IR-775 was not detectable in the LAA at 24 hours after surgery, but at 48 hours after surgery it was detectable in the LAA, and it was more concentrated at the ablation line than in other parts of the heart (Figure S7). The fluorescein intensity of LAA IR-775 was ranked in the following order: RFCA+L-IR-775 $>$ RFCA+IR-775 $>$ SO+L-IR-775 $>$ blank (Figure 2A and B). The fluorescein intensity of IR-775 in the LAA was higher than in any other organs in the RFCA+LIR-775 group (Figure 2C and D).

\section{Cardiac electrophysiological examination}

Representative electrophysiological recordings and the rates of electrical conduction block in four different phases are shown in Figure 3 and Table S1. Before ablation
(Phase 1), pacing electrocardiogram can be detected in all groups. At 5 minutes after ablation (Phase 2), pacing electrocardiogram could not be detected in all groups. At 1 week (Phase 3) and 1 month after ablation (Phase 4), pacing electrocardiogram could not be detected in most rabbits in the RFCA+L-DOX group, while it was detectable in most rabbits in the RFCA group that did not receive L-DOX. Since all of the rabbits injected with free DOX only survived for a maximum of 48 hours due to acute toxicity, a pacing electrocardiogram could not be performed at later times in these animals.

\section{L-DOX tissue distribution}

The concentration of L-DOX in the LAA and in other parts of the heart was roughly the same on the first day after surgery. However, on the second day after surgery, the concentration of L-DOX in the LAA was higher than that in the other parts of heart. More interestingly, the 

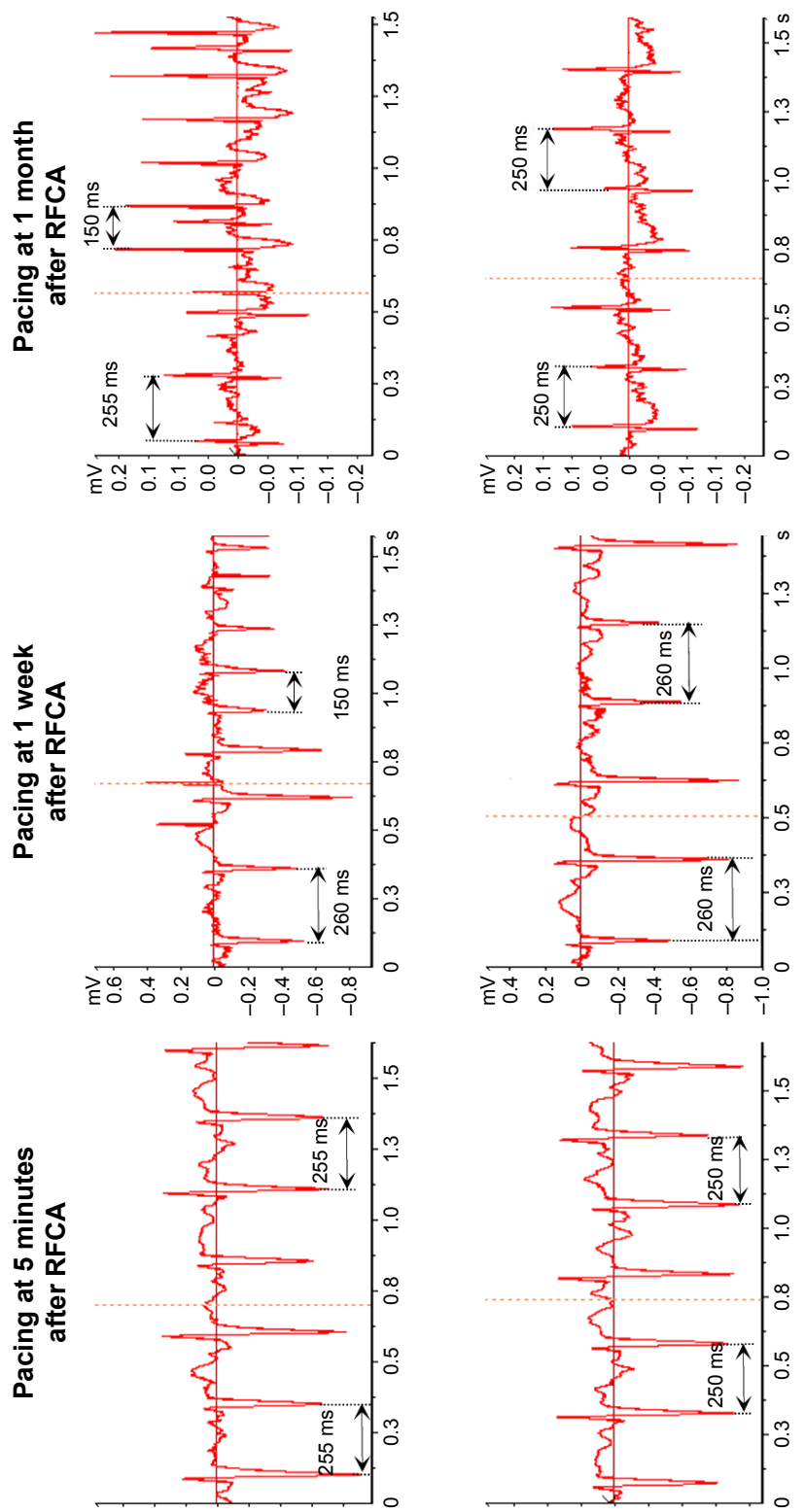

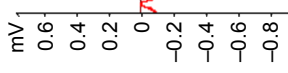
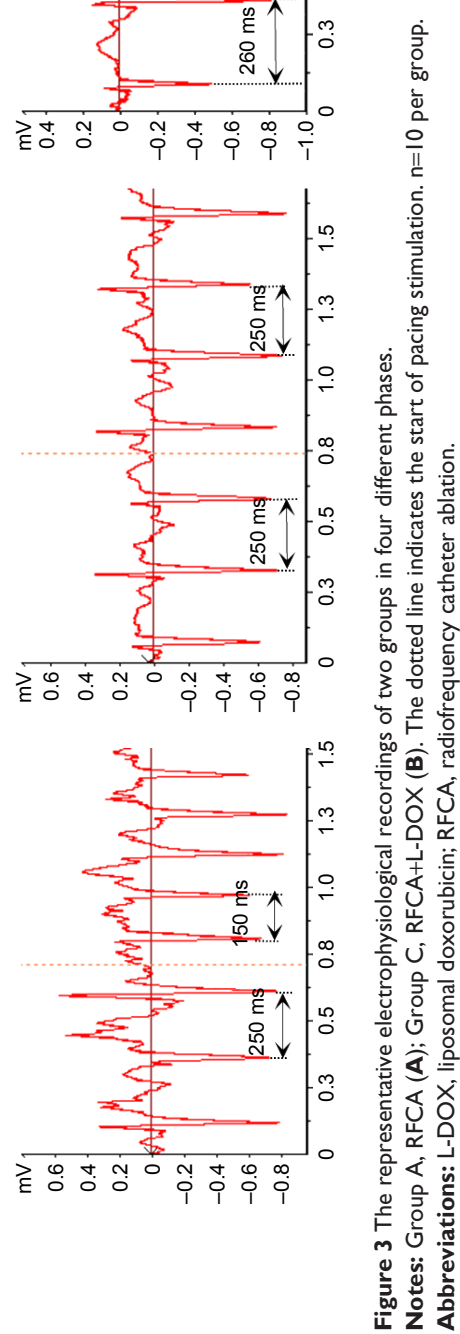
concentration of L-DOX at the LAA ablation site on the fifth and the seventh day after surgery was significantly higher than in other parts of the heart or in other organs. Liposomes remained in the liver and spleen for a long time, likely due to the ability of liposomes to passively target the liver and spleen. However, concentrations of L-DOX in lung and kidney were significantly lower than that in other organs (Figure 4A). On the second day after surgery, compared with free DOX, liposomes increased the content of DOX in the LAA ablation area and significantly reduced the DOX content in other parts of the heart and kidney (Figure 4B). The drug targeting index (DTI) of L-DOX for each organ is shown in Table S2. On the second day after surgery, the DTI of the LAA was the highest, and was significantly higher than that of the heart.
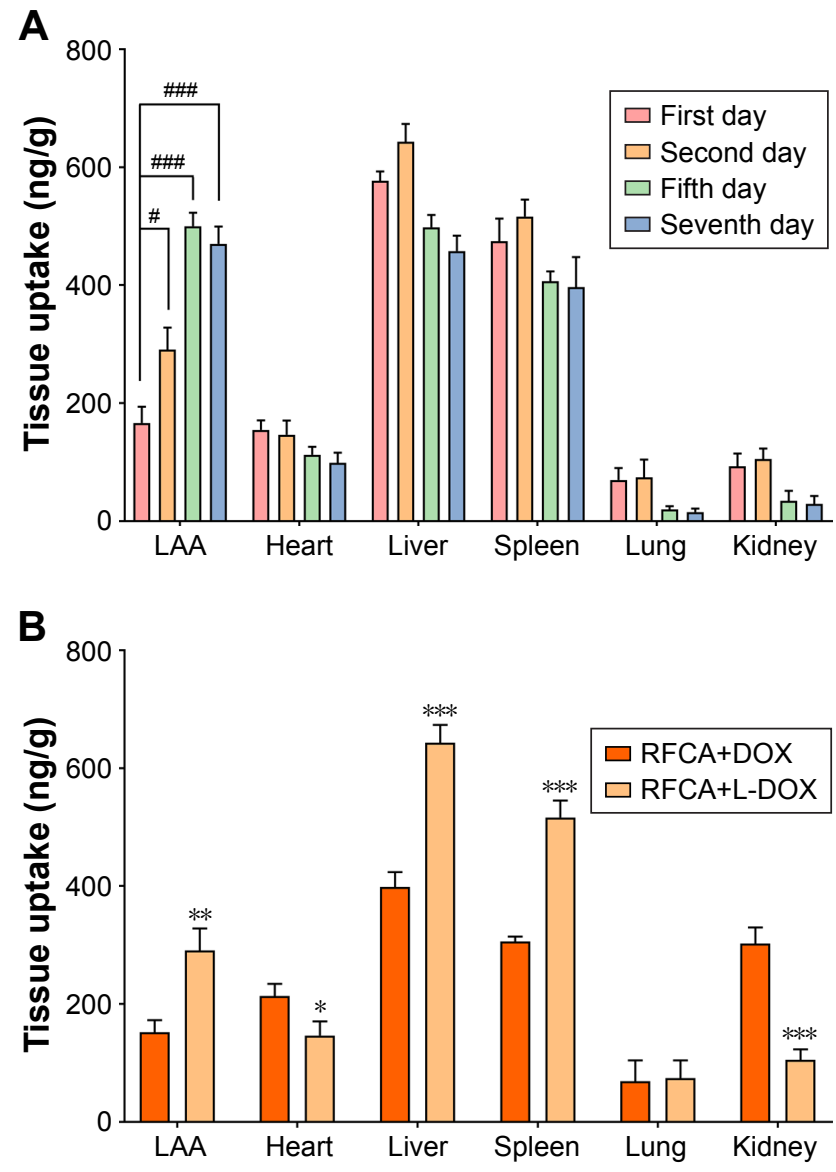

Figure 4 Plots of bio-distribution.

Notes: (A) Plots of DOX bio-distribution in rabbits receiving RFCA+L-DOX. The rabbits were sacrificed on different days after intravenous drug administration, as indicated. (B) Plots of DOX bio-distribution in rabbits in the RFCA+DOX group and RFCA+L-DOX group on the second day after intravenous drug administration. "Heart" refers to the other parts of the heart aside from the LAA. ${ }^{P}<0.05$, \#\#P $P<0.00$ I vs I day after intravenous administration. ${ }^{*} P<0.05,{ }^{*} * P<0.0$ I, $* * * P<0.00$ I vs free DOX treatment. $n=5$ per group, data are presented as means $\pm S D$.

Abbreviations: DOX, doxorubicin; L-DOX, liposomal doxorubicin; LAA, left atrial appendage; RFCA, radiofrequency catheter ablation.

\section{Detection of LAA cardiomyocyte apoptosis}

Figure S8 shows the TUNEL assay findings. The percentage of TUNEL-positive cells in the LAA slides was more significantly increased in the RFCA+L-DOX group $(85.25 \% \pm 4.31 \%)$ than in the RFCA group $(63.03 \% \pm 5.51 \%$, $P<0.01)$ or the SO group $(6.36 \% \pm 1.61 \%, P<0.001)$.

\section{Western blot analysis of LAA proteins}

Western blot assays were utilized to detect protein levels of Bcl-2, Bax, and cleaved CASP3. Bcl-2 protein content in the RFCA+L-DOX group $(0.32 \pm 0.10)$ was lower than that in the RFCA group $(0.69 \pm 0.18, P<0.01)$. Bax protein content in the RFCA+L-DOX group $(1.87 \pm 0.11)$ was significantly higher than that in the RFCA group $(1.22 \pm 0.19, P<0.01)$. Cleaved CASP3 protein content in the RFCA+L-DOX group (1.76 \pm 0.14$)$ was significantly higher than that in the RFCA group $(1.20 \pm 0.15, P<0.001)$. However, the difference between the RFCA group and the SO group was substantially less apparent than the difference between the RFCA+L-DOX group and the SO group (Figure 5).

\section{Semi-quantitative IHC examinations}

IHC localization of the apoptotic markers Bcl-2, Bax, and cleaved CASP3 was assessed in the LAA. The levels of Bcl-2 in the RFCA+L-DOX group were significantly decreased relative to the $\mathrm{SO}$ group and RFCA group, whereas the Bax and cleaved CASP3 protein levels were significantly increased $(P<0.001)$. L-DOX treatment significantly downregulated $\mathrm{Bcl}-2$ expression while up-regulating $\mathrm{Bax}$ and cleaved CASP3 expression $(P<0.001)$. Nevertheless, no significant differences were detected in the expression of these three proteins after treatment between the RFCA group and the SO group (Figure 6).

\section{Evaluation of kidney and liver function}

Compared with the SO group, hepatic enzyme activities (ALT, AST) and serum renal product biomarkers (BUN, serum creatinine) were not significantly altered in RFCA group. Significant increases in levels of ALT, AST, BUN, and serum creatinine were observed in rabbits on the first 3 days in the RFCA+L-DOX groups in a dose-dependent manner. However, after the third day, the levels of those indicators began to decline (Figure 7).

\section{Echocardiography examination}

The results of echocardiographic examination demonstrated that RFCA+L-DOX treatment significantly reduced LVEF 
A

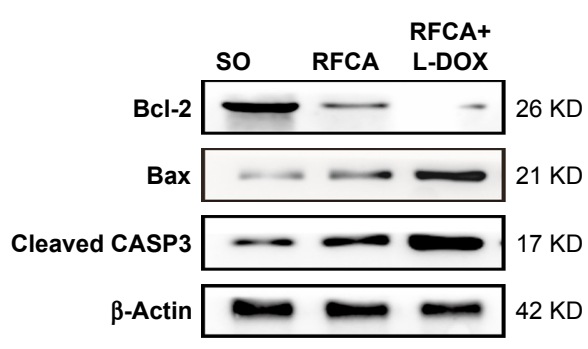

C

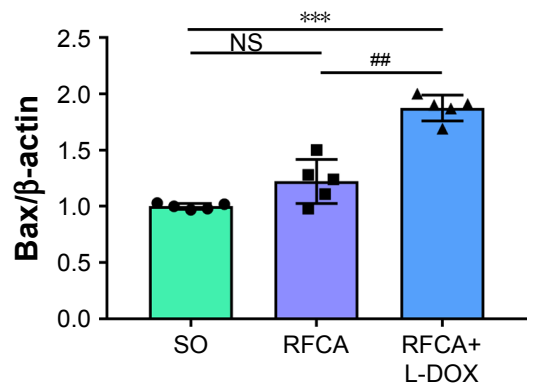

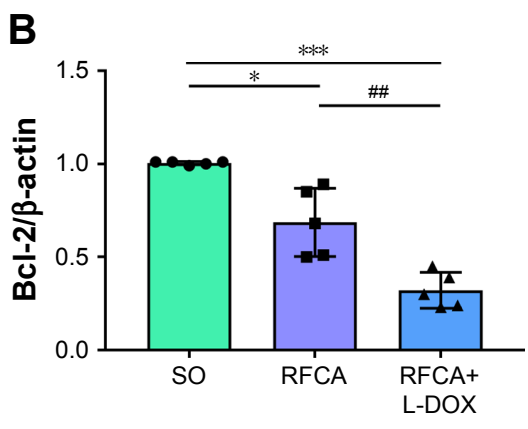

D

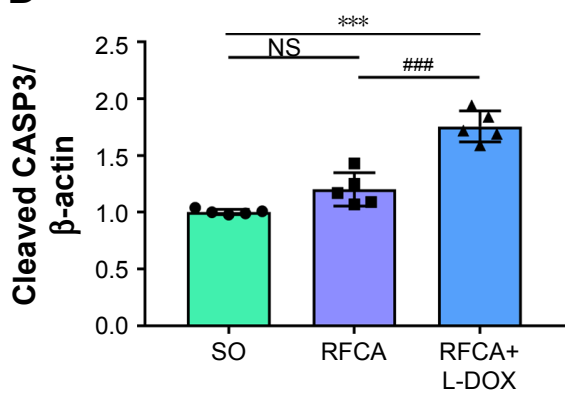

Figure 5 Expression level of apoptosis markers.

Notes: (A) Western blotting results for markers of apoptosis in different groups. Quantitative measurements of the band intensities of Bcl-2 (B), Bax (C), and cleaved CASP3 (D) are shown, following normalization to $\beta$-actin. ${ }^{*} P<0.05$, ${ }^{* * *} P<0.00$ I vs $S O$ treatment; ${ }^{\#} P<0.0$ I, ${ }^{\#} P<0.00$ I vs RFCA treatment. $n=5$ per group, data are presented as means \pm SD.

Abbreviations: L-DOX, liposomal doxorubicin; NS, not significant; RFCA, radiofrequency catheter ablation; SO, sham-operated.

A

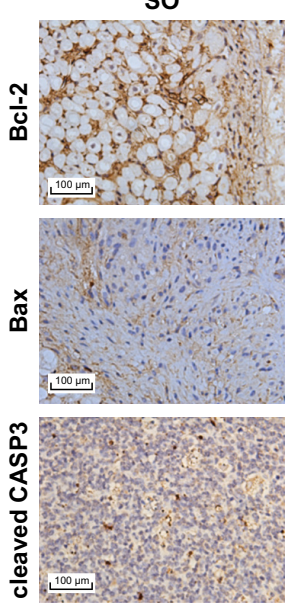

C

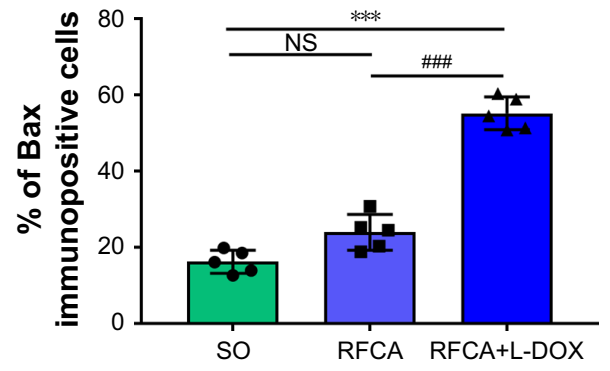

RFCA+L-DOX
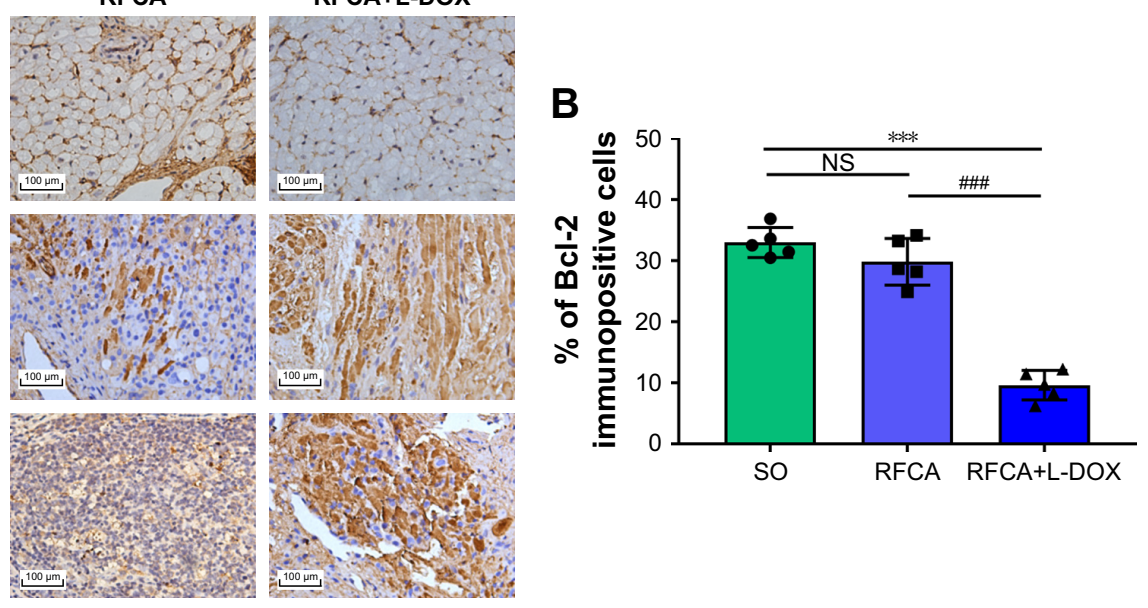

D

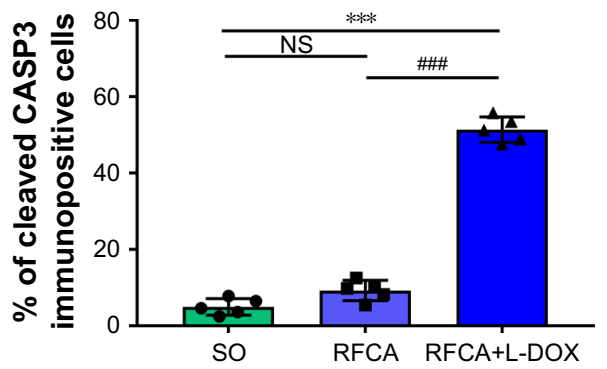

Figure 6 The expression of Bcl-2, Bax, and cleaved CASP3 in different groups (10×40).

Notes: (A) Representative Bcl-2, Bax, and cleaved CASP3 protein levels in different treatment groups were measured via immunohistochemistry analysis. (B-D) Bcl-2, Bax, and cleaved CASP3 protein expression in each group. ${ }^{*} * P<0.00$ I vs $S O$ treatment; ${ }^{\prime \prime} P<0.00$ I vs RFCA treatment. $n=5$ per group, data are presented as means \pm SD. Abbreviations: L-DOX, liposomal doxorubicin; NS, not significant; RFCA, radiofrequency catheter ablation; SO, sham-operated. 
A

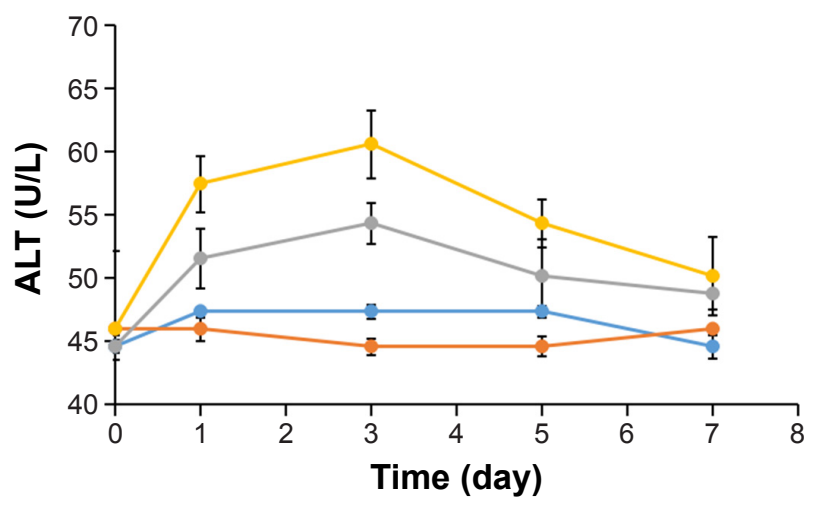

C

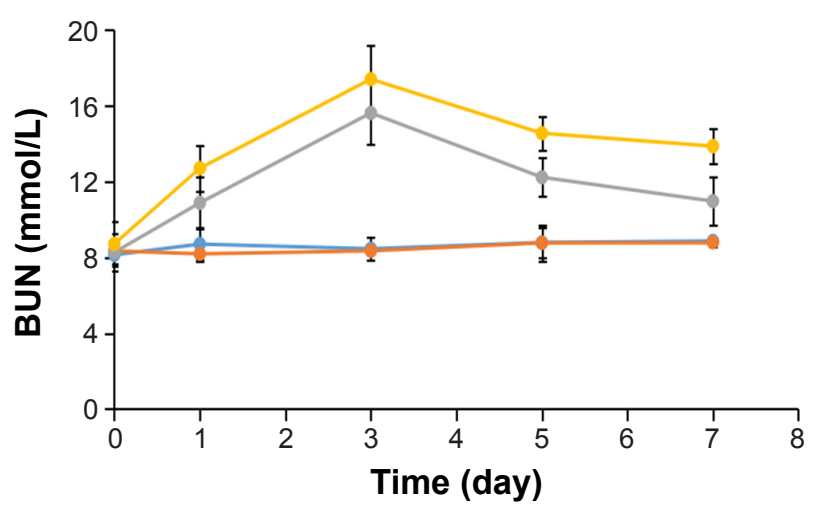

B

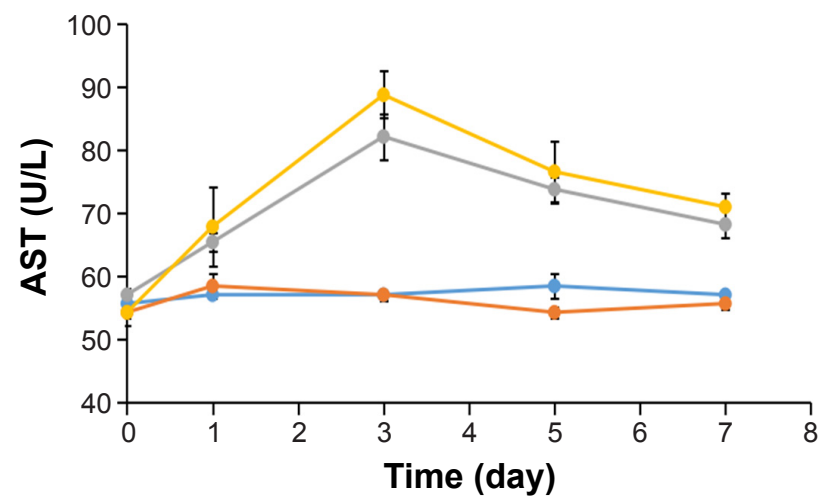

D

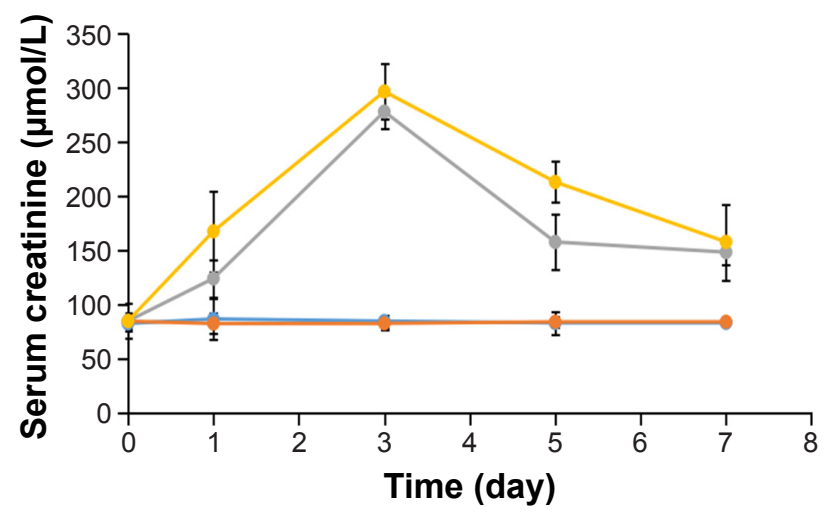

$\rightarrow \mathrm{SO} \rightarrow \mathrm{RFCA} \longrightarrow \mathrm{RFCA}+\mathrm{L}-\mathrm{DOX}(3 \mathrm{mg} \mathrm{DOX} / \mathrm{kg}) \multimap \mathrm{RFCA}+\mathrm{L}-\mathrm{DOX}(4 \mathrm{mg} \mathrm{DOX} / \mathrm{kg})$

Figure 7 Liver and kidney function markers in the serum of different treatment groups.

Notes: Levels of ALT (A), AST (B), BUN (C), and serum creatinine (D) in each treatment group. $n=5$ per group, data are presented as means \pm SD.

Abbreviations: BUN, blood urea nitrogen; DOX, doxorubicin; L-DOX, liposomal doxorubicin; RFCA, radiofrequency catheter ablation; SO, sham-operated.

and LVFS compared with RFCA treatment on the seventh day. In contrast, LVEDD, stroke volume, LVPWD, and LVEDV were increased in RFCA+L-DOX group (Figure S9).

\section{Discussion}

We made the following observations in this study. First, we demonstrated that fluorescent liposomes (L-IR-775) are targeted to the LAA after RFCA. Second, we demonstrated that similar liposomes were able to target DOX to the LAA ablation sites. Third, L-DOX was shown to reduce the recovery of electrical conduction after RFCA. Fourth, L-DOX induced cardiomyocyte apoptosis at the LAA ablation line. Fifth, L-DOX in combination with RFCA of LAA almost had no serious adverse effects in rabbits.

At 48 hours after surgery, L-IR-775 was targeted to the LAA. We postulated that RFCA of the LAA induced an inflammatory reaction, enabling the inflammation-targeting of the fluorescently-labeled liposomes which were then phagocytosed by local macrophages. In the SO+L-IR-775 group, the IR-775 fluorescent signal in the LAA was very weak. This may be a result of the thoracotomy, which may have induced pericardial inflammation and limited L-IR-775 targeting of the inflammatory site. The IR-775 fluorescein intensity in the LAA in the RFCA+L-IR-775 group was higher than that in the RFCA+IR-775 group because L-IR775 had superior inflammation-targeting compared to the IR-775 solution. In summary, these results showed that the LAA-targeting ability of L-IR-775 was closely associated with ablation injury.

We found that electrical conduction failed to recover at 1 week or 1 month after ablation in the majority of rabbits in the RFCA+L-DOX group, whereas it did recover in most rabbits in RFCA group. RFCA injury is thermally mediated, resulting in coagulative tissue necrosis. As the heating of tissue is reduced with increasing distance from the catheter tip, cells in regions peripheral to the necrotic area may undergo heating insufficient to cause necrosis, but sufficient to provoke acute cell damage. ${ }^{18}$ This may explain the electrical 
conduction recovery after RFCA and long-term recurrence after AF ablation. The combination of L-DOX and RFCA in our study appeared to be more effective in mediating a permanent conduction block. This targeted therapeutic effect may be useful in reducing recurrence of AF after ablation.

The concentration of L-DOX in the LAA on the second day after surgery was higher than that in other regions of the heart. This suggests that the liposomes began to target the LAA and changed the distribution characteristics of DOX in vivo. In addition, L-DOX has a strong affinity for the liver, spleen, and especially LAA ablation sites where many macrophages are present. The accumulation of L-DOX in the ablation site of LAA also gradually increased over time. This may be as a result of liposomes increasing DOX circulation in vivo, and being able to subsequently target the inflamed sites created by RFCA. ${ }^{19}$

Our positive TUNEL testing results in the RFCA group are consistent with the data reported by Winston et al, ${ }^{20}$ who investigated the periphery of the RF lesion in the myocardial tissue of pigs. However, some investigators have observed no detectable induction of apoptosis at the periphery of RF lesions in the rat myocardium. ${ }^{18}$ The TUNEL method aims to detect DNA strand breaks that occur during apoptosis. Nonetheless, necrosis induced by RFCA can also produce DNA breaks and thus may allow for TUNEL staining. Although the TUNEL test was clearly positive at the ablation site, additional testing including Western blotting and immunohistochemistry were consistently negative in the RFCA group. Our study did find, however, that L-DOX administration markedly aggravated cardiomyocyte apoptosis after RFCA. L-DOX could thus kill the remaining cardiomyocytes in the ablation area and transition zone of the LAA, thus overcoming the previously incomplete RFCA.

L-DOX produced a transient, reversible injury to the liver and kidney in rabbits in a dose-dependent manner. We observed decreased LVEF in both the RFCA+L-DOX group and the RFCA group compared with the SO group, but a more pronounced decrease in LVEF was observed in the former compared to the latter. In addition to cardiac "depressive" effects associated with anesthesia and thoracotomy, the cardiotoxicity of DOX might have contributed to the decreased LVEF. The optimal dose of DOX must therefore be investigated in our future studies.

Since L-DOX such as DOXIL ${ }^{\circledR}$ and MYOCET $^{\circledR}$ have been marketed and used clinically, this paper may yield lots of clinical applications. For instance, after RFCA surgery, the commercially available L-DOX can be administered to AF patients, and it is expected that L-DOX can increase the apoptosis of cardiomyocytes in the ablated area and transition zone in order to reduce long-term recurrence after RFCA. Nevertheless, this clinical application will have to be tested first because DOX is an agent with cardiotoxicity and its adverse effects on cardiac function cannot be avoided; thus, the study of effective dose may face challenges when ensuring safety.

We acknowledge several limitations. First, DOX is a potent anti-tumor agent with cardiotoxicity. Although liposomes are used as carriers for DOX, the adverse effects of DOX on cardiac function cannot be avoided, especially when using a large dose, as we did in this study. We hope that in our further studies, the minimum effective dose can be obtained and the side effects on the heart can be avoided as much as possible. Second, radiofrequency ablation combined with another, safer nano-formulation requires further investigation. Third, we call for further study in additional models before translating to clinical trials.

\section{Conclusion}

In this study, an L-DOX formulation with potential inflammation-targeting properties was successfully developed, and its potential use for LAA-targeting after circumferential ablation around the anterior and posterior wall of the LAA was investigated. The present results provide evidence that L-DOX reduces the recovery of electrical conduction after RFCA due to L-DOX-induced apoptosis of cardiomyocytes in the ablated area and transition zone of the LAA.

\section{Acknowledgments}

We would like to thank the Science and Technology Commission of Shanghai Municipality and the National Natural Science Foundation of China for supporting this study. This work was supported by grants from the Science and Technology Commission of Shanghai Municipality (16140901400 and 18140903500) and the National Natural Science Foundation of China (81870245).

\section{Disclosure}

The authors report no conflicts of interest in this work.

\section{References}

1. Kirchhof P, Benussi S, Kotecha D, et al. 2016 ESC Guidelines for the management of atrial fibrillation developed in collaboration with EACTS. Eur Heart J. 2016;37(38):2893-2962.

2. Kalla M, Sanders P, Kalman JM, Lee G. Radiofrequency catheter ablation for atrial fibrillation: approaches and outcomes. Heart Lung Circ. 2017;26(9):941-949.

3. Verma A, Jiang CY, Betts TR, et al. Approaches to catheter ablation for persistent atrial fibrillation. N Engl J Med. 2015;372(19):1812-1822. 
4. Kuck KH, Brugada J, Fürnkranz A, et al. Cryoballoon or radiofrequency ablation for paroxysmal atrial fibrillation. NEngl J Med. 2016;374(23): 2235-2245.

5. Yagishita A, Sparano D, Cakulev I, et al. Identification and electrophysiological characterization of early left atrial structural remodeling as a predictor for atrial fibrillation recurrence after pulmonary vein isolation. J Cardiovasc Electrophysiol. 2017;28(6):642-650.

6. Sawhney N, Anousheh R, Chen W, Feld GK. Circumferential pulmonary vein ablation with additional linear ablation results in an increased incidence of left atrial flutter compared with segmental pulmonary vein isolation as an initial approach to ablation of paroxysmal atrial fibrillation. Circ Arrhythm Electrophysiol. 2010;3(3):243-248.

7. Cappato R, Negroni S, Pecora D, et al. Prospective assessment of late conduction recurrence across radiofrequency lesions producing electrical disconnection at the pulmonary vein ostium in patients with atrial fibrillation. Circulation. 2003;108(13):1599-1604.

8. Ouyang F, Antz M, Ernst S, et al. Recovered pulmonary vein conduction as a dominant factor for recurrent atrial tachyarrhythmias after complete circular isolation of the pulmonary veins: lessons from double lasso technique. Circulation. 2005;111(2):127-135.

9. Bänsch D, Bittkau J, Schneider R, et al. Circumferential pulmonary vein isolation: wait or stop early after initial successful pulmonary vein isolation? Europace. 2013;15(2):183-188.

10. Koyama T, Sekiguchi Y, Tada H, et al. Comparison of characteristics and significance of immediate versus early versus no recurrence of atrial fibrillation after catheter ablation. Am J Cardiol. 2009;103(9): 1249-1254.

11. Ichikawa Y, Ghanefar M, Bayeva M, et al. Cardiotoxicity of doxorubicin is mediated through mitochondrial iron accumulation. J Clin Invest. 2014;124(2):617-630.
12. Howard MD, Hood ED, Zern B, Shuvaev VV, Grosser T, Muzykantov VR. Nanocarriers for vascular delivery of anti-inflammatory agents. Annu Rev Pharmacol Toxicol. 2014;54(1):205-226.

13. Desimone CV, Prakriti BG, Tri J, et al. A review of the relevant embryology, pathohistology, and anatomy of the left atrial appendage for the invasive cardiac electrophysiologist. J Atr Fibrillation. 2015;8(2):1129.

14. Hocini M, Shah AJ, Nault I, et al. Localized reentry within the left atrial appendage: arrhythmogenic role in patients undergoing ablation of persistent atrial fibrillation. Heart Rhythm. 2011;8(12):1853-1861.

15. Sadek MM, Maeda S, Chik W, et al. Recurrent atrial arrhythmias in the setting of chronic pulmonary vein isolation. Heart Rhythm. 2016;13(11): 2174-2180.

16. Yan JD, Xin H, Zheng YJ, Liu W, Gao N, Huang HB. Examine the ADM level in the serium and the tissue by fluorescence spectrophotometry. Chin J Lab Diagn. 2007;5:596-597.

17. Liang YJ, Xp W, Shi Z. Determining doxorubicin concentration in nude mice xenograft by fluorescence spectrometry. Chin J Clin Pharmacol. 2005;4:296-298.

18. Dos Santos LF, Antonio E, Serra A, et al. Radiofrequency ablation does not induce apoptosis in the rat myocardium. Pacing Clin Electrophysiol. 2012;35(4):449-455.

19. Zhuge Y, Zheng ZF, Xie MQ, Li L, Wang F, Gao F. Preparation of liposomal amiodarone and investigation of its cardiomyocyte-targeting ability in cardiac radiofrequency ablation rat model. Int J Nanomedicine. 2015;11:2359-2367.

20. Winston B, Nalesnik M, Bazaz R. TUNEL assay for identifying ablated myocardium. Pacing Clin Electrophysiol. 2010;33(12):1548-1549.
International Journal of Nanomedicine

\section{Publish your work in this journal}

The International Journal of Nanomedicine is an international, peerreviewed journal focusing on the application of nanotechnology in diagnostics, therapeutics, and drug delivery systems throughout the biomedical field. This journal is indexed on PubMed Central, MedLine, CAS, SciSearch $®$, Current Contents $\AA /$ Clinical Medicine,

\section{Dovepress}

Journal Citation Reports/Science Edition, EMBase, Scopus and the Elsevier Bibliographic databases. The manuscript management system is completely online and includes a very quick and fair peer-review system, which is all easy to use. Visit http://www.dovepress.com/ testimonials.php to read real quotes from published authors. 